\title{
Reconstrucción de la pared anterior del tórax: a propósito de una nueva alternativa
}

\section{Anterior chest wall reconstruction-a new alternative}

\author{
Mauricio Velásquez Galvis, MD ${ }^{1,2}$ Juan Carlos Varón, MD ${ }^{3}$
}

\section{Resumen}

La resección y posterior reconstrucción de la pared anterior del tórax por patologías neoplásicas o benignas representa un verdadero reto para el cirujano de tórax. Es imperativo en estos casos un trabajo multidisciplinario en el que están involucradas muchas especialidades médicas y no médicas para brindar el mejor resultado en términos de salud, bienestar, aspecto cosmético y futuro oncológico en el caso que así sea.

Se ha descrito una gran variedad de materiales para estos procesos reconstructivos impulsando a los cirujanos a utilizar su creatividad con el objetivo de resolver diferentes retos en el momento de la cirugía.

En este artículo se describe una serie de 4 casos, en los que la reconstrucción del esternón se pudo realizar con PEEK $^{\mathrm{TM}}$ diseñado a la medida para cada paciente. Se describe el caso clínico, las características quirúrgicas y el desenlace.

Palabras clave: reconstrucción de la pared del tórax, osteosíntesis, prótesis, tumor esternal.

\begin{abstract}
Removal and subsequent reconstruction of the anterior chest wall as a result of benign or neoplastic conditions is a real challenge for the cardiothoracic surgeon. In these cases, it is imperative to undertake a multidisciplinary task involving many medical and non-medical specialties to provide the best outcome in terms of health, well-being, cosmetics and oncology future, if appropriate.
\end{abstract}

A wide range of supplies for these reconstructive processes has been described encouraging surgeons to use their creativity to solve different challenges at the time of surgery.

This paper discusses a series of 4 cases, in which sternal reconstruction could be performed with custom-designed PEEK ${ }^{\mathrm{TM}}$. Clinical case material, surgical characteristics and outcomes are described.

Keywords: chest wall reconstruction, internal fixation, prosthesis, sternal tumor.
${ }^{1}$ Departamento de Cirugía, Servicio de Cirugía de Tórax, Fundación Valle del Lili, Hospital Universitario. Cali, Colombia.

${ }^{2}$ Facultad de Ciencias de la Salud, Universidad ICESI. Cali, Colombia.

${ }^{3}$ Cirugía de Tórax, Clínica CEHOCA. Santa Marta, Colombia.

Autor de correspondencia Mauricio Velásquez. Correo electrónico: mauriciovelasq@yahoo.com. Recibido: 10/7/2018. Aceptado: 10/8/2018. 


\section{Introducción}

La resección y posterior reconstrucción de la pared anterior del tórax por patologías neoplásicas o benignas representa un verdadero reto para el cirujano de tórax. En estos casos se combina la necesidad de restablecer la función y la estructura, pero, además, la necesidad de brindar un resultado cosmético adecuado teniendo en cuenta los principios oncológicos cuando está indicado. Es entonces cuando el trabajo multidisciplinario es primordial, lo cual le agrega una mayor complejidad a todo el proceso (1).

Se ha descrito una gran variedad de materiales para estos procesos reconstructivos impulsando a los cirujanos a utilizar su creatividad con el objetivo de resolver diferentes retos en el momento de la cirugía. Mallas de diferentes materiales (politetrafluoroetileno [PTFE], mallas sintéticas y mallas absorbibles) barras metálicas y de metil-metacrilato son algunos de los materiales más frecuentemente descritos para corregir el defecto que deja la resección, además del proceso que se debe seguir cubriendo el defecto con tejido nativo por medio de colgajos miocutáneos (1-3).

El PEEK ${ }^{\mathrm{TM}}$ (poliéter éter cetona) es un polímero técnico termoplástico semicristalino, que tiene un bajo coeficiente de fricción, alta resistencia al uso y al desgaste, y es biológicamente inerte, por lo que ha tenido una gran acogida en ortopedia, neurocirugía y cirugía maxilofacial para reconstruir defectos importantes por pérdida ósea (4).

En este artículo se describe una serie de 4 casos, realizados entre enero de 2016 y mayo de 2017, en los que la reconstrucción del esternón se pudo realizar con PEEK $^{\text {TM }}$ diseñado a la medida para cada paciente. Se tuvo un trabajo colaborativo intenso entre la industria médica, el cirujano y el paciente para obtener un resultado muy superior al que se podría obtener con los materiales y técnicas anteriores a este desarrollo.

\section{Casos clínicos}

\section{Caso 1}

Paciente mujer de 58 años con diagnóstico de cáncer de seno izquierdo en 2014 que fue llevada a una mastectomía con vaciamiento ganglionar axilar y te- rapia hormonal con buena evolución. 1 año después presentaba dolor torácico que se manejó sintomáticamente con el concurso de la clínica del dolor, pero se tornó intratable, por lo que se amplió la evaluación. Se realizó una tomografía por emisión de positronestomografía computarizada (PET-CT), la cual mostró un compromiso metastásico de todo el esternón y parcialmente de la primera y segunda costillas izquierdas, por lo que se remitió a cirugía de tórax. Se discutió el caso en una junta de oncología y se decidió que la paciente se beneficiaba de una resección de todo el esternón comprometido y una reconstrucción personalizada. En la Figura 1 se observa el esternón protésico en PEEK $^{\mathrm{TM}}$ luego de la resección oncológica del esternón y fijado a la pared torácica.

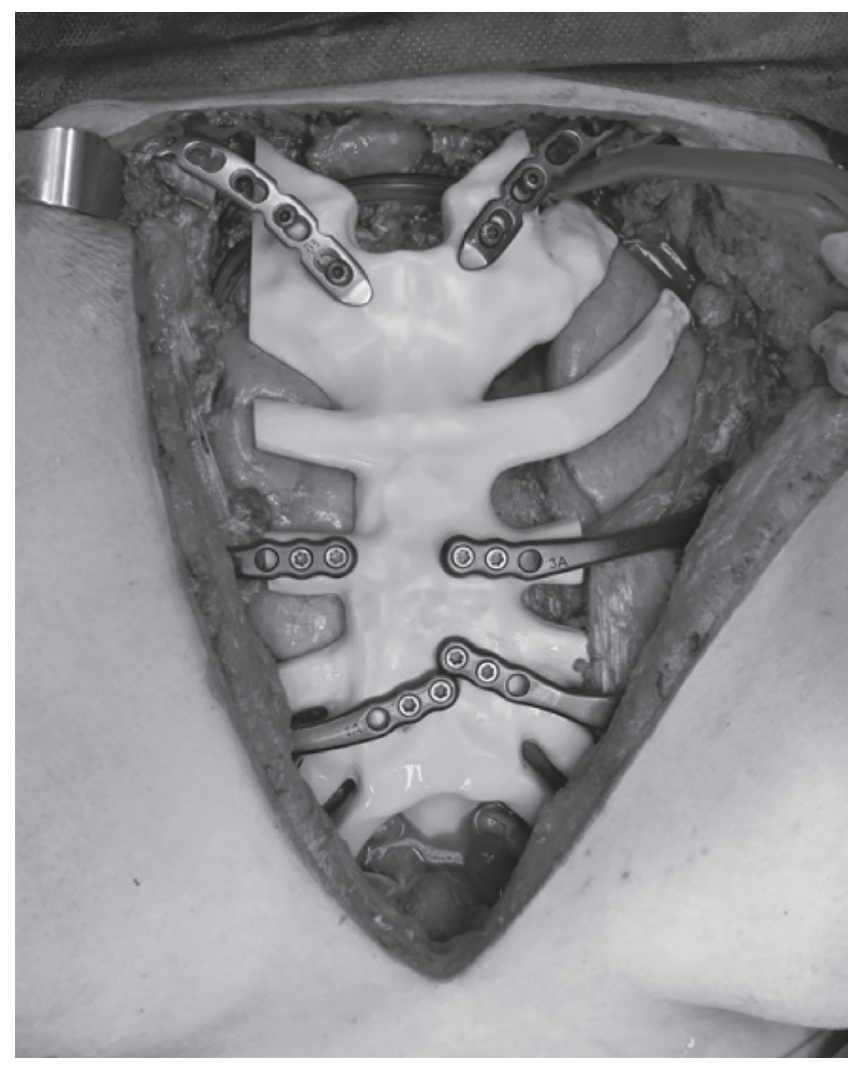

Figura 1. Prótesis esternal en PEEK ${ }^{\mathrm{TM}}$ fijada a la pared torácica anterior.

\section{Caso 2}

Paciente hombre de 42 años con un antecedente de trauma cerrado de tórax hace más de 20 años que solo requirió observación sin alguna patología detectada. Desde hace 10 años presenta dolor esternal, múltiples 
consultas médicas y tratamientos sintomáticos sin mejoría definitiva, finalmente se le realizó una resonancia magnética nuclear de la pared torácica que demostró una pseudoartrosis de la unión manubrio-esternal y, en vista de la sintomatología, se decidió llevar a cirugía para resecar este segmento comprometido y hacer una reconstrucción personalizada (Figura 2).

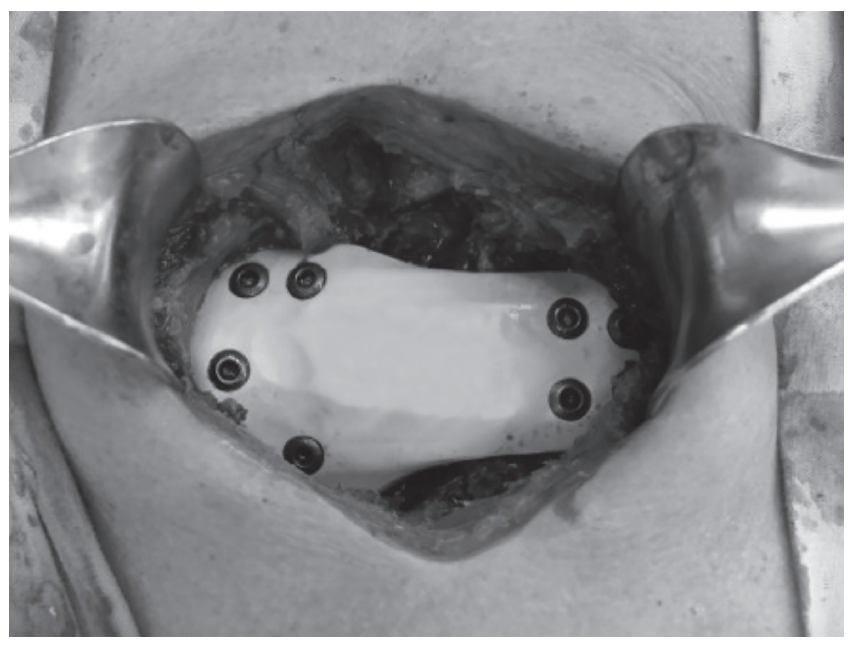

Figura 2. Imagen intraoperatoria que muestra al fondo la ausencia del segmento de la unión manubrio-esternal y la prótesis de PEEK ${ }^{\mathrm{TM}}$ estabilizando el esternón.

\section{Caso 3}

Paciente mujer de 12 años, que proviene de una zona rural muy apartada del país, quien presentaba ausencia del esternón desde el nacimiento, por lo que había sido evaluada por diferentes especialidades médicas durante todo el transcurso de su vida sin que se le presentase una solución definitiva. Finalmente se remitió al servicio de cirugía torácica y se decidió planear y realizar una reconstrucción del esternón de forma personalizada. En las Figuras 3 y $\mathbf{4}$ se observa durante la cirugía la ausencia del esternón y luego la prótesis en PEEK $^{\mathrm{TM}}$ fijada a la pared torácica con la que se reconstruye la pared anterior del tórax.

\section{Caso 4}

Paciente hombre de 57 años quien presentó la aparición de una masa no dolorosa en la región esternal hace 20 años, la cual tuvo un crecimiento acelerado en los últimos meses (Figura 5). Se realizó una tomografía de tórax que mostró una masa de $20 \times 15 \mathrm{~cm}$ dependiente del esternón con un crecimiento externo predominantemente y múltiples áreas de calcificación, sin observarse signos radiológicos que sugieran la infiltración a otras estructuras ni cavidades. Se descartó una extensión tumoral a distancia y posteriormente se decidió realizar una resección tumoral en bloque con la reconstrucción esternal personalizada (Figura 6). La patología mostró un condrosarcoma de bajo grado histológico con bordes de resección libres de infiltración tumoral.

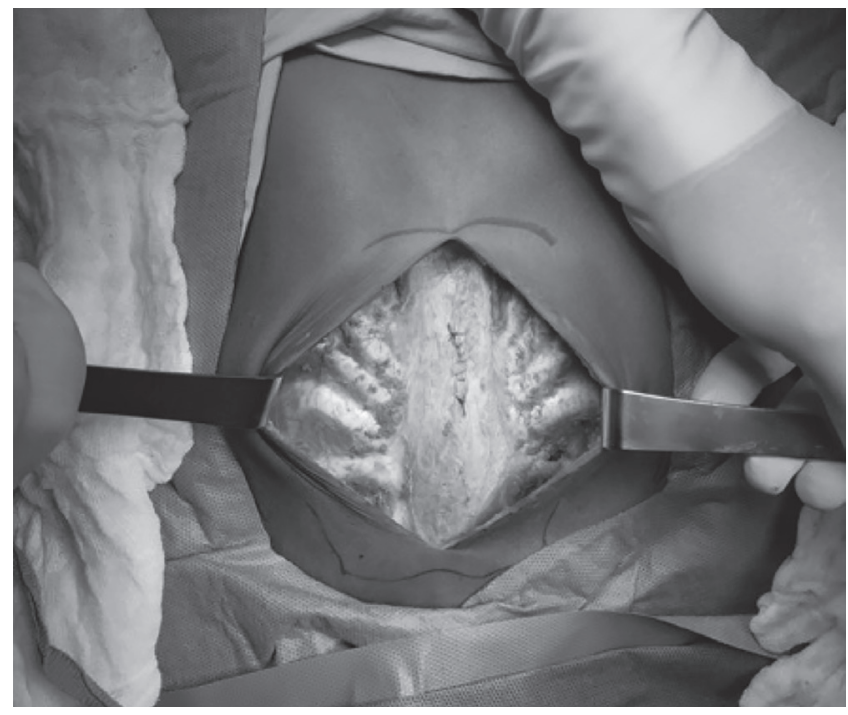

Figura 3. Imagen intraoperatoria que demuestra la ausencia de esternón.

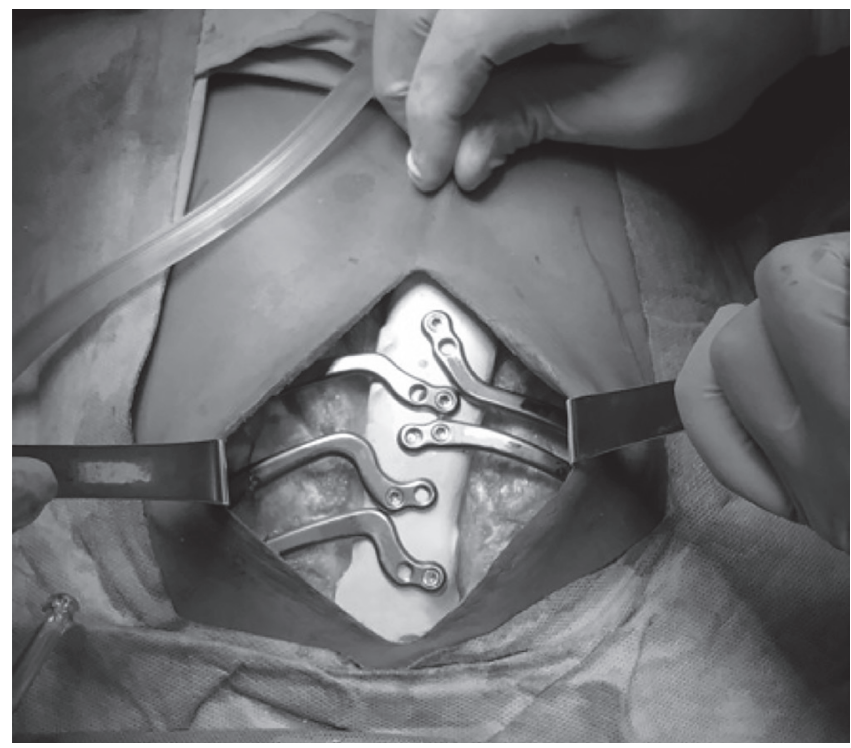

Figura 4. Imagen intraoperatoria que muestra la prótesis de $\mathrm{PEEK}^{\mathrm{TM}}$ que reconstruye la pared anterior del tórax. 


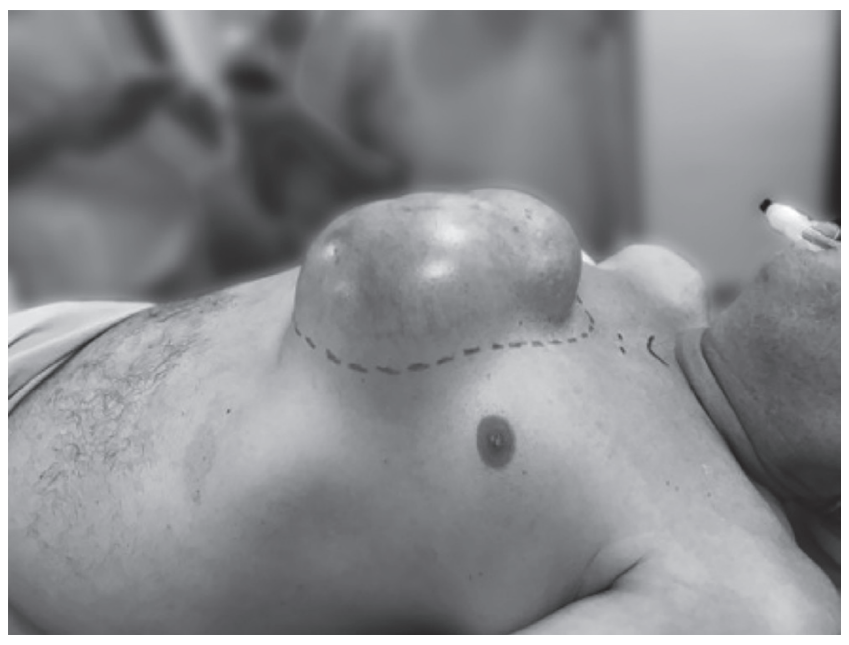

Figura 5. Imagen del paciente con un gran tumor en la pared anterior del tórax.

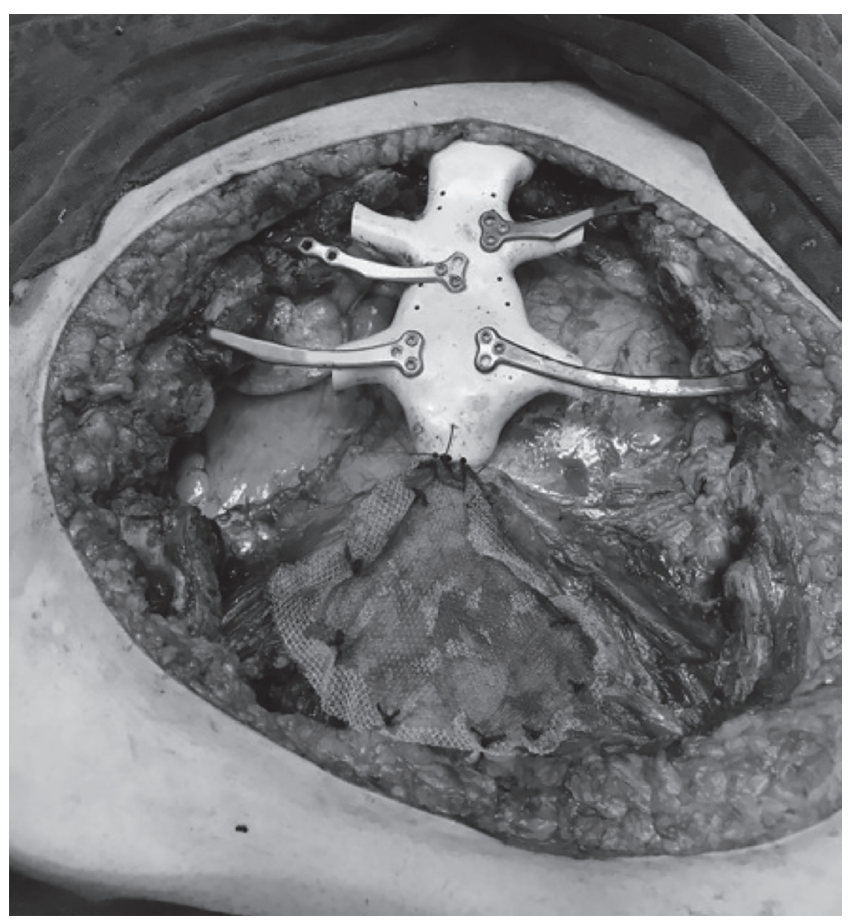

Figura 6. Imagen intraoperatoria del paciente luego de la resección del gran tumor de la pared anterior del tórax y la reconstrucción con la prótesis de PEEK ${ }^{\mathrm{TM}}$.

\section{Discusión}

El interés por nuevas alternativas en el proceso de la reconstrucción de la pared torácica ha sido creciente, tanto por las dificultades y riesgos de complicaciones por los materiales que se han usado tradicionalmente como por el interés en aportar unas soluciones más funcionales a nivel fisiológico y estructural. El desa- rrollo tecnológico de software y de impresoras 3-D ha permitido que la unión de la industria médica y los cirujanos pueda proveer estas soluciones en diferentes materiales que son completamente fabricadas para un paciente especifico, lo que permite la realización de cirugías más precisas y con reconstrucciones mucho mejores que acortan el tiempo quirúrgico porque evitan la manipulación y moldeo de los implantes en el acto operatorio (5).

El concepto de biocompatibilidad ha venido evolucionando y refinándose hasta llegar a definirse como el proceso mediante el cual un material desarrolla las funciones internas para las cuales se diseñó sin provocar una respuesta negativa en el organismo. En la actualidad, cualquier material que se vaya a utilizar para reemplazar un tejido humano debe tener estudios de biocompatibilidad. También es importante entender que la biocompatibilidad es un proceso de doble vía, es decir que comprende los efectos del material en el organismo y también los efectos del organismo sobre el material. El PEEK ${ }^{\mathrm{TM}}$ es un polímero biocompatible que mantiene sus propiedades mecánicas a altas temperaturas, resistente a la fricción y al ataque orgánico e inorgánico de químicos y solventes, y que se ha venido utilizando de manera creciente en áreas como la neurocirugía y la cirugía maxilofacial con excelentes resultados; sin embargo, hasta ahora no se había mostrado su uso en cirugía torácica (4).

Las reconstrucciones de la pared anterior del tórax han sido siempre un gran reto para el cirujano torácico y su equipo puesto que en la resección de la lesión se comprometen ambos hemitórax, frecuentemente se deben resecar músculos que participan en la respiración (pectoral, esternocleidomastoideo) y se deben proveer resultados funcionales, oncológicos y cosméticos (1-3).

En 2011 se publicó un trabajo en el que, mediante un proceso complejo de moldes y utilización de diferentes materiales, se fabricaron intraoperatoriamente las prótesis hechas a la medida del paciente, demostrando resultados muy buenos en 101 pacientes, con presencia de infección en 15 pacientes y necesidad de retiro del material solo en 7 pacientes (6).

En 2014 iniciaron los reportes, empezando por uno de Turquía y otro de Francia $(7,8)$, donde mediante la pla- 
neación preoperatoria se fabricó a la medida del paciente el material, en estos casos de titanio, que se va a utilizar para la reconstrucción, lo cual permitió una resección más amplia, con menor tiempo operatorio y resultados mejores desde el punto de vista oncológico, funcional y cosmético. De forma similar se encuentran otros reportes más recientes en España que enfatizan en el diseño de la prótesis, lo que permite un poco más de flexibilidad durante los movimientos respiratorios $(9,10)$.

En nuestros casos se utilizó un material diferente, supremamente liviano, con biocompatibilidad similar al titanio, pero que por ser un polímero, le agrega ventajas durante la cirugía, pues este material permite el corte y la perforación intraoperatoriamente, lo que hace el proceso más versátil con la posibilidad de corregir cualquier error de cálculo de la planeación de la prótesis; además, su costo es menor al de los materiales previamente usados y los resultados cosméticos y funcionales son similares.

Al igual que en el trabajo de Aranda y colaboradores, la planeación que se realiza para la fabricación de la prótesis genera unos moldes que se pueden utilizar en el momento de la cirugía para definir los límites de resección del tumor, lo que agiliza aún más el proceso de la cirugía y la reconstrucción.

\section{Conclusión}

La reconstrucción de la pared anterior del tórax es un gran reto para el cirujano torácico. Aunque los nuevos materiales disponibles y los nuevos desarrollos de aplicación de estos materiales para las reconstrucciones torácicas toman un tiempo importante en la planeación, tienen enormes ventajas en el momento de la cirugía con resultados antes inimaginables, como se pudo observar en los casos presentados. Se requieren más estudios prospectivos con un mayor número de pacientes para poder evaluar con mayor precisión esta forma de reconstruir la pared anterior del tórax.

\section{Referencias}

1. Ferraro P, Cugno S, Liberman M, Danino MA, Harris PG. Principles of chest wall resection and reconstruction. Thorac Surg Clin. 2010;20(4):465-73.

2. Rocco G. Anterior chest wall resection and reconstruction. Oper Tech Thorac Cardiovasc Surg. 2013;18(1):32-41.

3. Thomas PA, Brouchet L. Prosthetic reconstruction of the chest wall. Thorac Surg Clin. 2010;20(4):551-8.

4. Toth JM, Wang M, Estes BT, Scifert JL, Seim HB 3rd, Turner AS. Polyetheretherketone as a biomaterial for spinal applications. Biomaterials. 2006;27(3):324-34.

5. Ng CS. Recent and future developments in chest wall reconstruction. Semin Thorac Cardiovasc Surg. 2015;27(2):234-9.

6. Girotti P, Leo F, Bravi F, Tavecchio L, Spano A, Cortinovis U, et al. The "rib-like" technique for surgical treatment of sternal tumors: lessons learned from 101 consecutive cases. Ann Thorac Surg. 2011;92(4):1208-15.

7. Turna A, Kavakli K, Sapmaz E, Arslan H, Caylak H, Gokce HS, et al. Reconstruction with a patient-specific titanium implant after a wide anterior chest wall resection. Interact Cardiovasc Thorac Surg. 2014;18(2):234-6.

8. Demondion P, Mercier O, Kolb F, Fadel E. Sternal replacement with a custom-made titanium plate after resection of a solitary breast cancer metastasis. Interact Cardiovasc Thorac Surg. 2014;18(1):145-7.

9. Aranda JL, Jiménez MF, Rodríguez M, Varela G. Tridimensional titanium-printed custom-made prosthesis for sternocostal reconstruction. Eur J Cardiothorac Surg. 2015;48(4):e92-4.

10. Moradiellos J, Amor S, Córdoba M, Rocco G, Vidal M, Varela A. Functional Chest Wall Reconstruction With a Biomechanical Three-Dimensionally Printed Implant. Ann Thorac Surg. 2017;103(4):e389-e391. 